\title{
Discovery of Common Putative Drug Targets and Vaccine Candidates for Mycobacterium tuberculosis sp.
}

\author{
Nalamolu Ravina Madhulitha, Pasala Chiranjeevi, Katari Sudheer Kumar and Amineni Umamaheswari* \\ Bioinformatics Centre, Department of Bioinformatics, SVIMS University, Tirupati-517507, India, Phone number: 08772287727
}

\begin{abstract}
Mycobacterium tuberculosis is the bacteria that cause tuberculosis (TB), an infection that usually affects the lungs and can be fatal without proper treatment. Combating through available drugs became a difficult task due to drug resistance and lack of appropriate common targets against genetically diverse strains. Since to improve efficacy, the effective targets should be identified and critically assessed. In the study, we aim to predict the potential novel targets against $M$. tuberculosis strains by employing in silico approach. The complete proteomic datasets of 23 . tuberculosis strains was comparatively processed by executing R-scripts and eventually predicted 3906 'conserved gene products'. Further, we performed subtractive proteomic approach in search of promising crucial targets. Consequently, eight enzymes and two membrane proteins were prioritized as new therapeutic and vaccine targets respectively which found to have more interactors in network with high-confidence score, druggability and antigenicity. Therefore, outcomes of the study emphasize the importance of new targets may counteract with falsepositive/negatives and facilitate appropriate potential targets for a new insight of reliable therapeutic development.
\end{abstract}

Keywords: Mycobacterium tuberculosis, Multidrug resistance tuberculosis and Extensive drug resistant tuberculosis.

Article Info: Received 18 Feb 2019; $\quad$ Review Completed 20 March 2019; $\quad$ Accepted 23 March 2019; $\quad$ Available online 15 April 2019

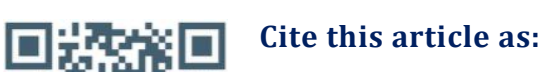

Nalamolu RM, Pasala C, Katari SK, Amineni U, Discovery of Common Putative Drug Targets and Vaccine Candidates for Mycobacterium tuberculosis sp. , Journal of Drug Delivery and Therapeutics. 2019; 9(2-s):67-71 http://dx.doi.org/10.22270/jddt.v9i2-s.2603

*Address for Correspondence:

Amineni Umamaheswari, Bioinformatics Centre, Department of Bioinformatics, SVIMS University, Tirupati-517507, India, Phone number: 08772287727

\section{INTRODUCTION}

Tuberculosis (TB) is one of the major infectious diseases affecting human beings caused by Mycobacterium tuberculosis. Tuberculosis is either latent or active, latent TB means the TB bacteria is in the body, but the body's defenses (immune system) are keeping it static from turning into active TB 1 . Latent TB also called inactive TB or TB infection isn't contagious. It can turn into active $\mathrm{TB}$, so treatment is important for the person with latent TB to prevent its conversion to active form. The symptoms of TB depend on the area of which the body has been infected; primary infection of tuberculosis might affect the lungs, pulmonary tuberculosis (PTB) complications include hemoptysis, pneumothorax, bronchiectasis, malignancy and chronic pulmonary aspergillosis 2. Symptoms of TB include fatigue, weight loss, and lack of appetite, chills, fever and night sweats. An extra pulmonary (EP) infection can affect any part of the body, the infection spreads through hematogenous route i.e., mainly through blood to the other sites of the body like urinary tract, genital tract, bones and joints etc ${ }^{3}$. Genital TB generally occurs as secondary to pulmonary (commonest) or extrapulmonary TB like gastrointestinal tract, kidneys, skeletal system, ISSN: 2250-1177 meninges and miliary TB through hematogenous and lymphatic route. Over $95 \%$ of new TB cases and deaths occur in developing countries with India accounting for $40 \%$ of the world's TB burden 4. Co-infection with human immunodeficiency virus (HIV) patients with more liberal immigration from high risk to low-risk areas due to globalization has been responsible for increased incidence all over the world 5 .

Globally, 10.4 million new cases of active TB were estimated among which 1.2 million HIV positive people are included, of the 3.5 million were women and 1.0 million were children. About 1,70,000 children have died of TB (excluding children with HIV) ${ }^{6}$. World Health Organization (WHO) recommends the patient should have six months of TB drug treatment (WHO 2015). This consists of a two month "intensive" treatment phase followed by a four-month "continuation" phase 7 . Improper treatment or interruption to medication uptake makes $M$. $t b$ resistance to drugs ${ }^{8}$. Adults with drugresistant TB should always receive an effective second-line injectable agent as part of their regimen. The emergence of antibiotic resistance $M$. tuberculosis around the world owes as a genetically more diverse form. The resistance mechanisms during evolution described to date are due to 
point mutations on the bacterial chromosome considerably results in phenotypic variations in $M$. tuberculosis strains. The prevalence of antibiotic resistance appears to be partly determined by geographical factors. Increase in multi-drug resistance (MDR) and extensively drug-resistance (XDR) were also reported in tuberculosis cases ${ }^{9}$.

Therefore, the WHO (2016) changed their recommendations on the use of drugs for the treatment of drug-resistant TB. However, adverse effects need to be carefully monitored while using injectable effective agents. For instance, hearing loss and nephrotoxicity are the most frequent severe side effects ${ }^{10}$.

Despite, Bacille Calmette-et-Guerin (BCG) vaccine provides minimal immunity or protection against TB; it is causing side effects like swollen lymph nodes, small red areas at the site of injection, fever, blood in urine, frequent or painful urination, upset stomach and vomiting ${ }^{11}$. To overcome the adverse, side effects and non-efficacious drugs and vaccines in treating and preventing TB caused by MDR and XDR strains, there is an emergent need to discover novel, potential drug targets and vaccine candidates for effective TB therapeutics design. Therefore, in the current study, we aim to predict novel potent drug targets and vaccine candidates against MDR and XDR M. tuberculosis strain through in silico approaches.

The experimental approaches are tedious and laborious obtain very few results hence computational approaches were used to identify the putative drug targets. Comparative proteomic approach, subtractive proteomic approach, metabolic pathway analysis, non-homologous gut flora analysis, domain search analysis, and protein network analysis are extensively used for the prediction of potential drug targets of the pathogen causing TB. In this scenario, potential targets must be essential for the growth and survival of the pathogen. Further designing of inhibitors should hinder the function exclusively the pathogen and should avoid the undesirable cross-reactivity with the human proteins. The availability of complete proteome sequences of the selected pathogen in combination with Bioinformatics tools and databases is of great importance in reducing the problem of searching for potential drug targets/vaccine candidates in a large pool of proteomes. Therefore, in the current study, we intended to explore potential novel therapeutic and vaccine candidates from numerous genetically diverse $M$. tuberculosis strains inhabitants around the globe for designing new reliable broad-spectrum inhibitors.

\section{MATERIALS AND METHODS}

\subsection{Comparative Proteomic Analysis and Dataset Preparation:}

We used proteomes of 23 virulent $M . t b$ strains in the current study, among them $M$. $t b_{-}$H37Rv strain was considered as a reference due to its predominant role in TB 12 . Complete proteomes were retrieved from the National Center for Biotechnology Information (NCBI), an imperative resource for enormous biological information 13. In comparative proteomic approach, data processing was done using inhouse bash and $\mathrm{R}$ scripts in Linux environment for downstream dataset preparation and quantification in order to get conserved (common) proteins ${ }^{14}$.

\subsection{Subtractive Proteomic Approach:}

Common protein sequences were subjected for non-human homology 15 by aligning each protein against human proteome for unidentical protein sequences or protein sequences with less than $30 \%$ identity.

\subsection{Gene Essentiality and Non-human Homology Analysis:}

The derived dataset of conserved proteins was carried to target screening using different channels that optimizes a large number of conserved proteins to few putative targets. In order to screen essential gene products from the dataset, essential gene analysis was performed using the Database of Essential Genes (DEG) accessed at http://tubic.tju.edu.cn/deg/ 15. Essential proteins were further assigned to non-homology analysis against human proteome ${ }^{16}$. The query sequence that did not match any hit better than the threshold (bit score of >100) were considered as ' $M$. tuberculosis-specific' protein. Protein alignments with an expectation value of (E- value) $<10^{-10}$ were considered as more significant hits. Such proteins were regarded as essential, based on the assumption that similar proteins that are essential in one organism are likely to be essential in another ${ }^{17}$.

\subsection{Analysis of Non-homology against Host:}

The aim of the non-homology analysis is to identify pathogen specific-proteins that are non-homologous to the host. The significance of this step is to minimize undesirable crossreactivity of the drug thereby preventing its binding to active sites of the host's homologous proteins. Essential proteins were further assigned to non-human homologous analysis, protein alignments E-value $>10^{-3}$ was considered as nonhuman homologous proteins as described in a previous study ${ }^{18}$. The aim of non-human homology analysis against host is to identify pathogen specific-proteins. The significance of this step is to minimize undesirable crossreactivity of the inhibitors with host proteins. The essential protein dataset was searched for non-host homology analysis to identify common proteins of $M$. $t b$ strains.

\subsection{Host-pathogen Metabolic Pathway Analysis:}

KEGG (Kyoto Encyclopaedia of Genes and Genome) accessed at http://www.genome.jp/pathways.html 19 is a pathway database used as a source of metabolic pathway information. Pathways which do not appear in the host but present in the pathogen according to KEGG database have been identified as pathways unique to $M$. tuberculosis. The corresponding protein sequences of enzymes involved in unique pathways were identified 20 . The pathways of drug targets were checked and the drug targets having alternative pathways were not considered because blocking of these drug targets would be ineffective as the product is synthesized by the alternative way.

\subsection{Non-homology Analysis against Human Gut-flora and Domains:}

All delineated proteins were sought out for similarity search with the proteome of human gut-flora taken from literature reports 21 to evade adverse reactions of chemical inhibitors on host microbiome. Further, the shortlisted enzymes were searched against homologous human domains in Pfam and SMART (Simple Modular Architecture Research Tool) databases to eliminate host's domain level similar protein sequences of MTB, which comprise a large collection of protein families. Finally, the selected enzymes that are unidentical to human proteins/domains were considered as putative targets for validation.

\subsection{Protein Interaction Network Analysis:}

Analysis of protein-protein networks of the derived target proteins was performed through STRING v10.0, a database providing critical assessment and integration of proteinprotein interactions, including direct (physical) as well as indirect (functional) associations ${ }^{22}$. The preferred high 
confidence interactions with score $\geq 0.700$ and not more than 50 interactors were set to acquire significant target proteins possess with higher interactomes in the networks as well as to avoid false positives and false negatives. The identified targets that are specific and important in various metabolic networks of the pathogen were considered as putative drug targets.

The confidence score of a target $=$

Number of interactants of target enzyme by used methods Total number of methods used

\subsection{Subcellular Localization Prediction:}

Cellular locations of the key target proteins were identified using PSORTb v3.0.2 [24]. Further validated with Subcellular localization of the identified drug targets could be used to obtain information about their potential functions. Subcellular location of the potential target differentiates cytoplasmic, periplasmic or inner membrane proteins that are metabolically important drug targets and outer membrane or extracellular proteins as vaccine candidates. Broad spectrum candidates were classified as either drug targets or vaccine candidates based on their subcellular localization using PSORTb and further validated with CELLO v2.5. The tools that employ four types of sequence coding schemes such as $\mathrm{ABC}$ transporter proteins and sequence composition based on physicochemical properties of amino acids. Moreover, the obtained surface exposed proteins were tested for their antigenicity using VaxiJen server for prediction of protective antigens, tumor antigens where antigenic proteins showing score more than the threshold (0.4) were predicted as good antigens and affirmed as subunit vaccines candidates.

\section{RESULTS AND DISCUSSION}

\subsection{Comparative and subtractive proteomic analysis:}

As shown in table (1), The common proteins from chromosomal and plasmid DNAs were preferred for target screening as being conserved among several strains could be useful in implementing a common inhibitor. Execution of Rscripts and codes could facilitate the proteome-wide comparison of total 92,325 proteins of 23 M. tuberculosis strains obtained 3906 M. tb specific common proteins. The dataset derived from the comparative and subtractive proteomic approach has been illustrated in Figure (1); composed of conserved proteins with $30-100 \%$ identity.

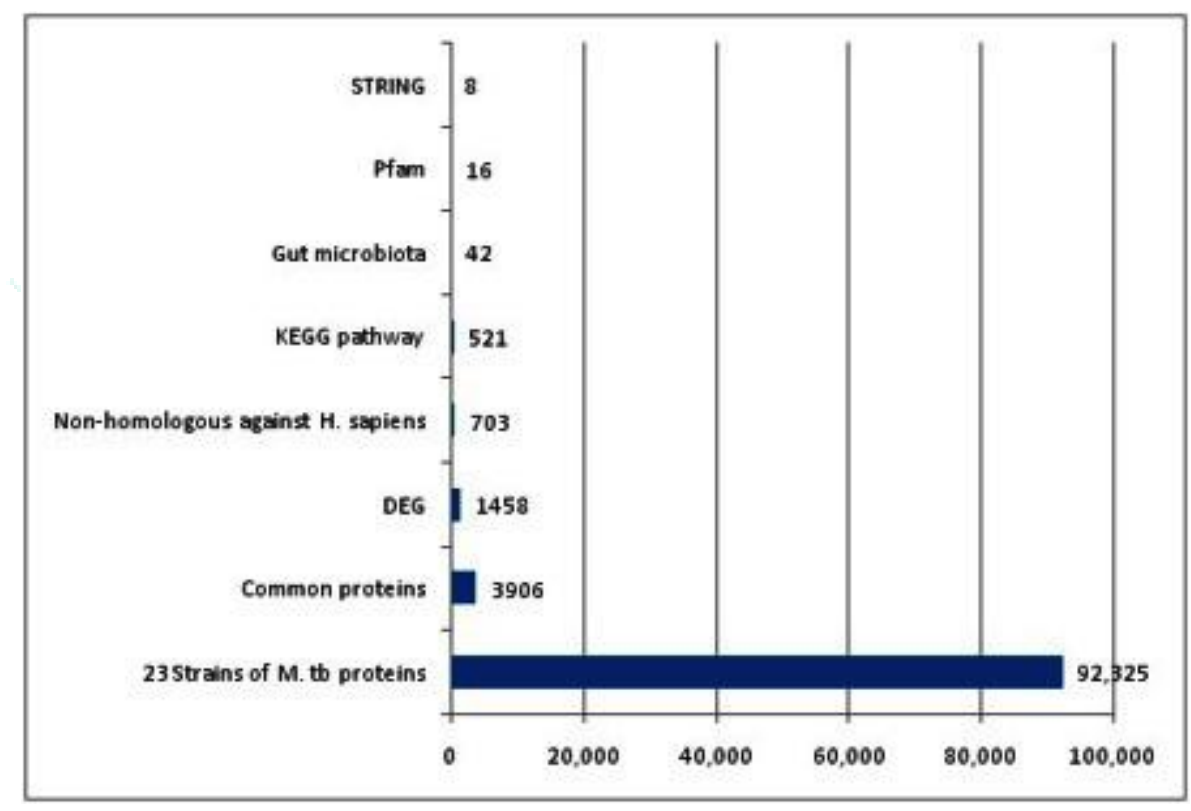

Figure 1: Comparative and subtractive drug target identification approach

\subsection{Gene essentiality and non-homology analysis:}

As shown in table (1), Essential genes are indispensable to maintain cellular life, as they are vital for replication, survival, and viability of the pathogen. Of the common proteins, about 1,458 essential proteins were derived by dint of essentiality analysis. Subsequently, a non-human homologous search of essential proteins had ensued 703 proteins were absent in host (human) and are of great concern in view of designing drugs absolutely specific for the pathogen.

Table 1: Identification of common Drug targets and vaccine candidate of M.tb strains

\begin{tabular}{|l|l|l|}
\hline No. & Analyzed criteria & Outcome from the target screening \\
\hline 1. & Proteins in 23 MTB strains & 92,325 \\
\hline 2. & Common proteins among 23 MTB strains & 3906 \\
\hline 3. & Predicted essential common genes products & 1458 \\
\hline 4. & Predicted non-human homologs & 703 \\
\hline 5. & Pathogen specific unique proteins and enzymes of pathway analysis & 521 \\
\hline 6. & Unique proteins without alternative pathway analysis & 282 \\
\hline 7. & Non-homologous to gut microbiota & 42 \\
\hline 8. & Non- homologous to human domains & 12 \\
\hline 9. & Proteins with high interaction networks & 8 \\
\hline 10. & Surface exposed membrane proteins after subcellular location & 2 \\
\hline
\end{tabular}




\subsection{Metabolic functional association and potential drug targets:}

As shown in table (1), Comparative metabolic pathway analysis of non-homologous proteins had unveiled 521 unique proteins comprises 239 enzymes and 282 nonenzymes including membrane proteins, which are absent in H. sapiens (distinct pathways). In the present study, metabolic pathways namely porphyrin and chlorophyll, Glycerophospholipid metabolism, arginine and proline metabolism, glyoxylate and dicarboxylate carbon metabolism, phenylalanine, tyrosine and tryptophan metabolism, carotenoid biosynthesis, streptomycin biosynthesis polyketide sugar unit biosynthesis, glutathione metabolism and carbon metabolism contributed to the final drug targets list derived from the utmost analyses .

As shown in table (2), Pathways which are not present in the Homo sapiens but present in the Mycobacterium are designated as unique pathways. Design and targeting inhibitors against these non-homologous sequences could be the better approach for the generation of new drugs. Thus total 8 unique metabolic pathways have been taken in $M$. tuberculosis.

Table 2: Proposed common drug targets for $M . t b$

\begin{tabular}{|c|c|c|c|c|}
\hline No. & Protein Name & $\begin{array}{l}\text { Pathway Essential } \\
\text { enzymes }\end{array}$ & Biological Process & Molecular Function \\
\hline 1. & $\begin{array}{l}\text { Protoheme IX farnesyl } \\
\text { transferase (2.5.1.36) }\end{array}$ & Porphyrin and chlorophyll & Heme Biosynthesis & Transferase \\
\hline 2. & $\begin{array}{l}\text { Phosphatidyl serine } \\
\text { decarboxylase proenzyme } \\
(4.1 .1 .36)\end{array}$ & $\begin{array}{l}\text { Glycerophospholipid } \\
\text { metabolism }\end{array}$ & $\begin{array}{l}\text { Lipid biosynthesis, lipid } \\
\text { metabolism, Phospholipid } \\
\text { biosynthesis, Phospholipid } \\
\text { metabolism }\end{array}$ & Decarboxylase, lyase \\
\hline 3. & $\begin{array}{l}1 \text { pyrroline- } 5 \text {-carboxylate } \\
\text { dehydrogenase (1.2.1.88) }\end{array}$ & $\begin{array}{l}\text { Arginine and proline } \\
\text { metabolism }\end{array}$ & $\begin{array}{l}\text { Proline catabolic process to } \\
\text { glutamate }\end{array}$ & Nucleotide binding \\
\hline 4. & $\begin{array}{l}\text { Putative isocitrate lyase } \\
\text { metabolism } \\
(4.1 .3 .1)\end{array}$ & $\begin{array}{l}\text { Glyoxylate and } \\
\text { dicarboxylate carbon }\end{array}$ & Isocitrate metabolic process & $\begin{array}{l}\text { Isocitrate lyase } \\
\text { activity }\end{array}$ \\
\hline 5. & $\begin{array}{l}\text { Phospho-2- dehydro-3- } \\
\text { deoxyheptonate aldolase } \\
(2.5 .1 .54)\end{array}$ & $\begin{array}{l}\text { Phenylalanine, tyrosine and } \\
\text { tryptophan }\end{array}$ & $\begin{array}{l}\text { Chorismate biosynthetic } \\
\text { process }\end{array}$ & $\begin{array}{l}\text { 3-deoxy-7- } \\
\text { phosphoheptulonate } \\
\text { synthase activity }\end{array}$ \\
\hline 6. & $\begin{array}{l}\text { Phytoene synthase } \\
(2.5 .1 .99)\end{array}$ & Carotenoid biosynthesis & $\begin{array}{l}\text { carotenoid biosynthetic } \\
\text { process }\end{array}$ & $\begin{array}{l}\text { Farnesyl-diphosphate } \\
\text { farnesyl transferase } \\
\text { activity }\end{array}$ \\
\hline 7. & $\begin{array}{l}\text { dTDP-glucose 4, 6-dehydratase } \\
(4.2 .1 .46)\end{array}$ & Streptomycin biosynthesis & $\begin{array}{l}\text { Polyketide sugar subunit } \\
\text { biosynthesis }\end{array}$ & Coenzyme binding \\
\hline 8. & $\begin{array}{l}\text { Isocitrate dehydrogenase } \\
(1.1 .1 .42)\end{array}$ & $\begin{array}{l}\text { Glutathione metabolism, } \\
\text { carbon metabolism }\end{array}$ & Isocitrate metabolic process & $\begin{array}{l}\text { Isocitrate } \\
\text { dehydrogenase } \\
\text { activity }\end{array}$ \\
\hline
\end{tabular}

\subsection{Gut microbiota analysis:}

As shown in table (1), Gut microorganisms benefit the host in many ways such as cleaning the energy from the fermentation of undigested carbohydrates, synthesizing vitamins, subsequent absorption of short-chain fatty acids, degrading xenobiotics etc. The human microflora helps in numerous ways which provide resistance to colonization by pathogenic bacteria and indigenous opportunists by influencing the host immune system. Drug designed should neither harm the host enzymes nor hinder the function of enzymes of the gut flora which leads to side effects. Disruption of gut microbiota results in the development of many numerous pathologies in human health. As a result of the human gut-flora analysis, about 42 enzymes out of 239 . So, if design a drug for these non-homologous proteins that do not hinder the functioning of the human gut microbiota enzymes.

\subsection{Domain search:}

The domain similarity search between M. tuberculosis and host ensured the elimination of the protein domains which are homologous to host. Domain analysis using the Pfam and SMART databases resulted out 8 enzymes.

\subsection{Protein network Analysis:}

The selected drug targets were examined by protein-protein interaction network analysis. The network analysis of 8 enzymes and 2 vaccine candidates was determined using the
STRING database. Interactions with a score of $>0.400$ and interactions with $<50$ interactors were regarded as potential drug targets. The analysis of STRING database showed that all the proposed drug targets showed interactions with the high confidence scores of 0.900 and with low confidence interactions less than 50 were incorporated in the interaction network. Each network gives a particular group of proteins. Hovering over each node will display its annotation and full details of the protein, here network nodes represent proteins. Each node in the network has its own importance. Clustering in STRING has two different parameters to cluster the proteins. KMEANS is the parameter which particularly specifies the number of clusters and MCL is the parameter that indirectly related to the precision of the clustering which is mentioned as 'inflation'. The nodes can be deleted and change in the clustering was noted. Upon deletion of each node manually clustering MCL parameter or KMEANS parameter decrease in clustering coefficient was observed and considered as a functionally important protein in the metabolic network. Hence, all the drug targets were considered as potential targets for the pathogen causing tuberculosis.

\subsection{Vaccine candidate:}

As shown in table (1), The subcellular localization prediction determined that 8 enzymes were located in cytoplasmic side and 2 were in the surface exposed membrane (OMP) proteins. From VaxiJen server, the shortlisted OMPs with a score more than the threshold (0.4)

CODEN (USA): JDDTAO 
were predicted as good antigens and affirmed as subunit vaccines candidates. Among the common 10 proteins, two vaccine candidates such as Molybdenum transport system permease protein (ModB) and phosphate transport system permease protein (pstc1) was identified as common vaccine candidates for MTB strains causing tuberculosis. Hence, the identification of vaccine candidates would be a promising attempt towards designing T-cell epitope-driven common subunit vaccine for preventing $M$. tuberculosis infections. Similarly, two novel vaccine candidates are membrane components with highly antigenic to host, essential for pathogenesis, cell adhesion and to protect M. tuberculosis from osmotic gradients. The implementation of therapeutics such as inhibitor or vaccines against these proposed novel targets may become single or common therapeutic for several M. tuberculosis strains and could be effective due to targets involvement in vital pathways. Therefore, the results of the study will highlight the proposed novel targets as more potential therapeutic targets.

\section{CONCLUSION}

Globally, the emergence of multi-drug resistance among pathogenic $M$. tuberculosis is a growing concern in the medical field. The development of new antimicrobial agents against unexplored drug targets is of major concern to curb these problems. This paper describes and appraises the uncovering set of potential targets that are typically conserved in $M$. tuberculosis strains which present a new opportunity for the experimental biologists to test. We identified the pool of eight common potential drug targets and two surface exposed membrane proteins provides a basis for computer-aided drug and vaccine design strategies lending credence to our approach. Development of powerful drug molecules via in silico to in vivo pipelines against these putative targets may become potential therapeutics to combat present and upcoming drug-resistant M. tuberculosis strains as these are conserved targets critically essential for pathogens, non-homologous to human and gut-flora at sequence to structural level. Therefore, the proposed targets from the study would bring new possibility in obstructing the biological functions essential for the growth and survival of M. tuberculosis infections.

\section{ACKNOWLEDGMENTS}

NRM acknowledges to ICMR, New Delhi, for supporting her with the Senior Research Fellowship (ISRM/11(21)/2017). PC acknowledges to ICMR, New Delhi, for supporting him with the Senior Research Fellowship (3/1/3/JRF2014/HRD-8). Authors are highly thankful to DBT, Ministry of Science and Technology, Govt. of India for providing infrastructure facility to SVIMS Bioinformatics Center (BT/BI/25/037/2012(BIF-SVIMST)).

\section{CONFLICTS OF INTEREST}

The authors declare no conflict of interest.

\section{REFERENCES}

1. Coker R, McKee M, Atun R, Dimitrova B, Dodonova E, Kuznetsov S, Risk factors for pulmonary tuberculosis in Russia: a case-control study. British Medical Journal, 2006; 332(7533):85-87.

2. World Health Organization (WHO) Global Tuberculosis Report 2013. Geneva: 2013.

3. Chang CC, Crane M, Zhou J, Mina M, Post JJ, Cameron BA, Lloyd AR, Jaworowski A, French MA, Lewin SR, HIV and co-infections. Immunological Reviews, 2013; 254(1):114-42.

4. Mak A, Thomas A, Del Granado M, Zaleskis R, Mouzafarova N, Menzies D, Influence of multidrug resistance on tuberculosis treatment outcomes with standardized regimens, American Journal of Respiratory and Critical Care Medicine, 2008; 178(3):306-312

5. Skrahin A, Ahmed R, Ferrara K, Autologous mesenchymal stromal cell infusion as an adjunct treatment in patients with multidrug and extensively drug-resistant tuberculosis: an open-label phase 1 safety trial, Lancet Respiratory Medicine, 2014; 2(2):108-22.

6. Caminero JA, Sotgiu G, Zumla A, Migliori GB, Best drug treatment for multidrug-resistant and extensively drugresistant tuberculosis, Lancet Infectious Diseases, 2010; 10:621-629.

7. Udani PM, BCG vaccination in India and tuberculosis in children: newer facets. Indian Journal of Pediatrics, 1994; 61:451-462.

8. Zheng H, Lu L, Wang B, Pu S, Zhang X, Zhu G, Shi W, Zhang L, Wang H, Wang S, Zhao G, Zhang Y, Genetic basis of virulence attenuation revealed by comparative genomic analysis of Mycobacterium tuberculosis strain H37Ra versus H37Rv, PLoS ONE, 2008; 3:23-75

9. Johnson TJ, Abrahante JE, Hunter SS, Hauglund M, Tatum FM, Maheswaran SK, Briggs RE, Comparative genome analysis of an avirulent and two virulent strains of avian Pasteurella multocida reveals candidate genes involved in fitness and pathogenecity. BMC Microbiology, 2013; 13-106.

10. Hema K, Priyadarshini V, Pradhan D, Munikumar M, Sandeep S, Pradeep N, Suchitra MM, Umamaheswari A, Identification of Putative Drug Targets and Vaccine Candidates for Pathogens Causing Atherosclerosis, Biochemistry and Analytical Biochemistry, 2015; 4:1-9.

11. Singh V, Mizrahi V, Identification and validation of novel drug targets in Mycobacterium tuberculosis. Drug Discovery Today, 2017; 22:503-509.

12. Sarkar M, Maganti L, Ghoshal N, Dutta C, In silico quest for putative drug targets in Mycobacterium tuberculosis molecular modeling of candidate enzymes from lipopolysaccharide biosynthesis pathway, Journal of Molecular Modeling, 2012; 18:1855-1866.

13. Umamaheswari A, Pradhan D, Hemanth KM, Computer aided subunit vaccine design against pathogenic Leptospira serovars, Interdisciplinary Sciences: Computational Life Sciences, 2012; 4:38-45.

14. Altschul SF, Gish W, Miller W, Myers E, Lipman DJ, Basic local alignment search tool, Journal of Molecular Biology, 1990; 15:403-10.

15. Sridhar S, Comparative Analyses of the Proteins from Mycobacterium tuberculosis and Human Genomes, Identification of Potential Tuberculosis Drug Targets, Gene, 2016; 36:12-54.

16. Madhukar P, Marcel A, David D, Keertan D, Maziarn D, Catharina CB, Ann Ginsberg, Soumya S, Melvin S, Halieyesus G, Dick M, Mario R. Tuberculosis, Springer Nature reviews 2016; 56:21-45.

17. Raman $\mathrm{K}$, Chandra N, Mycobacterium tuberculosis interactome analysis unravels potential pathways to drug resistance, BMC Microbiology, 2008; 8:234.

18. Mustafa A, In silico Analysis and Experimental Validation of Mycobacterium tuberculosis-Specific proteins and peptides of Mycobacterium tuberculosis for immunological diagnosis and vaccine development, Medical Principals and Practi, 2013; 43-51.

19. Chiranjeevi P, Chandra SRC, Sudheer KK, Ravina MN, Aparna RB, Amineni Umamaheswari, An in silico study: Novel targets for potential drug and vaccine design against drug resistant $\mathrm{H}$. pylori, Microbial Pathogenesis, 2018; 122:156-161.

20. Cohen T, Becerra MC, Murray MB, Isoniazid resistance and the future of drug-resistant tuberculosis, Microbial Drug Resistance, 2004; 10:280-5.

21. Marco P, Penny C, John T, Chris B, Ningze P, Kristoffer F, Goran C, Jody C, Andreas H, Liisa H, Erik L, Sean R, Alex B, The Pfam protein family's database, Nucleic Acids Research, 2012; 10:106-16.

22. Devasundaram S, Deenadayalan A, An In silico analysis of potential human $\mathrm{T}$ cell antigens from Mycobacterium tuberculosis for the development of subunit vaccines against tuberculosis, Immunological Investigations, 2014; 43:137-59. 